\title{
Review: the only effect for surgery plus antiobiotics in vesicoureteric reflux is fewer febrile urinary tract infections
}

Wheeler D, Vimalachandra D, Hodson EM, et al. Antibiotics and surgery for vesicoureteric reflux: a meta-analysis of randomised controlled trials. Arch Dis Child 2003;88:688-94.

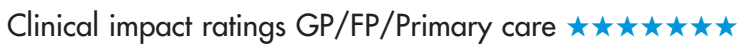

In children with vesicoureteric reflux (VUR), which treatments reduce urinary tract infection (UTI) and renal parenchymal injury?

\section{METHODS}

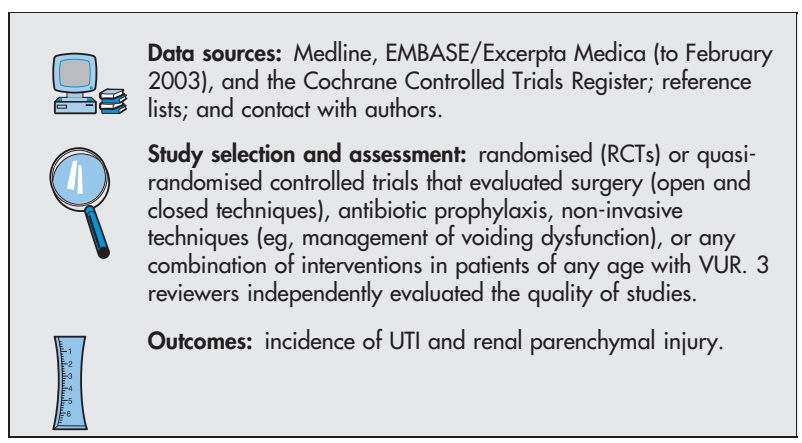

\section{MAIN RESULTS}

8 RCTs of 947 children $<15$ years of age were included. Studies originated in the US, Europe, and New Zealand. Antibiotic prophylaxis plus surgery $v$ antibiotics alone ( 7 RCTS). At 5 years, febrile UTI occurred less frequently in the combination treatment group than in the antibiotics alone group (table). The rates of UTI did not differ between groups at 2 or 5 years (table). Renal parenchymal injury did not differ between groups (table). Antibiotic prophylaxis $v$ no treatment ( 1 RCT). Groups did not differ for UTI (relative risk reduction [RRR] $75 \%, 95 \%$ CI -85 to 97 ) or renal parenchymal injury (RRR $60 \%$, CI -818 to 98$)$.

\section{CONCLUSION}

In patients with vesicoureteric reflux, surgery plus antibiotics reduces febrile urinary tract infection (UTI) more than antibiotics alone but does not reduce overall urinary tract infection or parenchymal injury.

For correspondence: Associate Professor $\mathrm{j}$ Craig, The Childreñs Hospital at Westmead, Sydney, New South Wales, Australia. jonc@pub.health.usyd. edu.au

Source of funding: Australian Kidney Foundation.

\section{Commentory}

he management of VUR has been based on the belief that children with VUR are at significant risk of further UTls leading to renal scarring and, on occasion, end stage renal disease. The metaanalysis by Wheeler et al, using the stringent criteria set by the Cochrane Collaboration, questions this view and adds to the growing uncertainty of the role of VUR in UTI and renal parenchymal injury. Taken together with studies showing that children without VUR are as likely to get renal scarring as those with VUR, it suggests that VUR is not the critical factor contributing to UTI related morbidity.

If this is the case, is the identification of VUR necessary? If VUR is not clinically important, it implies that a micturating cystourethrogram (MCU) should no longer be required for children presenting with UTI. Although this is an attractive proposition, the answer remains unknown because of a lack of adequately powered trials that compare treatment (either medical or surgical) with no treatment.

A study that addresses this important question is currently in progress, and the results will have a major impact on how children with UTIs are investigated. Until the results of this trial are available, the identification of VUR will remain important, especially for young infants. VUR is considered to be a risk factor for further UTIs, which are frequently associated with pyelonephritis in this group. Although not proven, identification of VUR and subsequent prophylactic treatment has the potential to decrease morbidity related to hospital admission and intravenous antibiotic treatment. In addition, an MCU remains a necessary investigation in male infants to exclude posterior urethral valves.

To summarise, the absence of appropriate evidence means that identification of VUR remains important in infants. For older children with non-febrile UTIs, surgical correction of VUR has no additional benefit when compared with antibiotics alone, implying that the identification of VUR is of limited clinical value.

Steven McTaggart, MD Queensland Child \& Adolescent Renal Service Brisbane, Queensland, Australia

Surgery and antibiotics $v$ antibiotics alone in children with vesicoureteric reflux ${ }^{*}$

\begin{tabular}{lllll}
\hline Outcomes & Number of RCTs & Weighted event rates & RRR (95\% CI) & NNT (CI) \\
\hline Febrile UTI at 5 years & 2 & $9.4 \% \vee 22 \%$ & $57 \%(30$ to 73$)$ & 8 (6 to 18) \\
UTI at 4-5 years & 3 & $36 \% \vee 37 \%$ & $1 \%(-26$ to 21$)$ & Not significant \\
\hline & & RRI (CI) & NNH \\
\hline UTI at 1-2 years & 4 & $25 \% \vee 20 \%$ & $6 \%(-45$ to 104$)$ & Not significant \\
$\begin{array}{l}\text { Renal parenchymal injury at 2 years } \\
\text { Renal parenchymal injury at 5 years }\end{array}$ & 2 & $60 \% v 26 \%$ & $80 \%(-83$ to 1768) & Not significant \\
\hline
\end{tabular}

${ }^{*} \mathrm{RCT}$ = randomised controlled trials; UTI = urinary tract infection. Other abbreviations defined in glossary; RRR, RRI, NNT, NNH, and Cl calculated from data in article. 